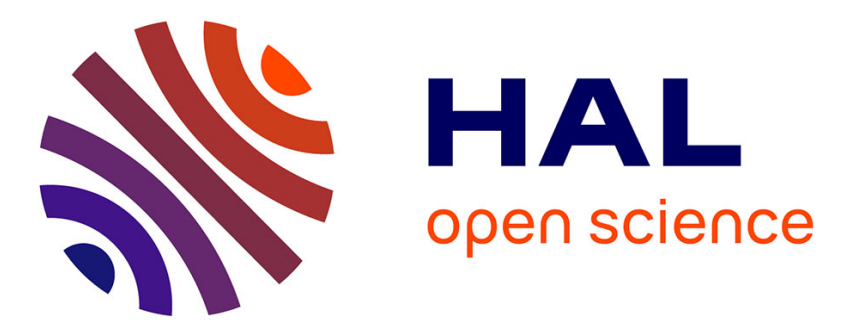

\title{
MAC design for 5G dense networks based on FBMC modulation
}

Rida El Chall, Benoit Miscopein, Dimitri Kténas

\section{To cite this version:}

Rida El Chall, Benoit Miscopein, Dimitri Kténas. MAC design for 5G dense networks based on FBMC modulation. 12th EAI International Conference on Cognitive Radio Oriented Wireless Networks (Crowncom 2017), Sep 2017, Lisbonne, Portugal. pp.310-321, 10.1007/978-3-319-76207-4_26 . cea01871426

\section{HAL Id: cea-01871426 https://hal-cea.archives-ouvertes.fr/cea-01871426}

Submitted on 10 Sep 2018

HAL is a multi-disciplinary open access archive for the deposit and dissemination of scientific research documents, whether they are published or not. The documents may come from teaching and research institutions in France or abroad, or from public or private research centers.
L'archive ouverte pluridisciplinaire $\mathbf{H A L}$, est destinée au dépôt et à la diffusion de documents scientifiques de niveau recherche, publiés ou non, émanant des établissements d'enseignement et de recherche français ou étrangers, des laboratoires publics ou privés. 


\title{
MAC design for 5G dense networks based on FBMC modulation
}

\author{
Rida El Chall, Benoit Miscopein, and Dimitri Kténas \\ CEA-Leti Minatec, 17 rue des Martyrs, 38054 Grenoble Cedex 9, France \\ Benoit.MISCOPEIN@cea.fr
}

\begin{abstract}
The fifth generation (5G) of wireless networks is currently under investigation in order to address the well-known challenges of the high capacity demands and traffic volume. The promising solutions to meet these targets can be achieved through ultra-densification, efficient use of spectrum and advanced filtered modulation techniques. In this paper, we present an enhanced MAC protocol for $5 \mathrm{G}$ small cells operating at $5 \mathrm{GHz}$ and assuming an FBMC physical layer. The proposed MAC design consists of scheduled-based and contention-based access schemes and involves a listen before talk (LBT) procedure to comply with ETSI regulations. The performance of the proposed FBMC-MAC design is then evaluated in dense deployment scenarios under different PHY/MAC parameter settings. Moreover, we study the performance of FBMC-MAC systems in the context of coexistence with WiFi systems.
\end{abstract}

Key words: 5G, FBMC, multiple access MAC design, LBT, LBE, dense small cell networks, contention access, scheduled access, CSMA/CA

\section{Introduction}

The ever increasing demand for higher data rate and reliable communications poses significant challenges for the existing wireless networks. Fifth generation (5G) cellular networks are expected to meet these intense demands as well as to support different kinds of applications and quality of service (QoS) requirements. Those included, enhanced mobile broadband, ultra-reliable and low-latency communications, and massive machine-type communications [1].

As far as capacity improvement is concerned, the common approaches are based on ultra-densification, exploitation of heterogeneous resources in available licensed, lightly-licensed and unlicensed spectrum bands; the spectrum resources being possibly used assuming traffic steering or aggregation mechanisms.

Moreover, relying on a modulation technique with higher efficiency than the one used in $4 \mathrm{G}$ is another key aspect of $5 \mathrm{G}$ network design. Among different possibilities, filter-bank multi-carrier (FBMC) modulation is considered as a candidate for $5 \mathrm{G}$ systems [2]. Indeed, FBMC through the use of well-designed prototype filters overcomes the limitations of the widely-used orthogonal frequency division multiplexing (OFDM) technique in terms of tight timing and frequency synchronization requirements. It can provide a very sharp frequency confinement of the signal which translates into a better spectrum utilization. 
In this paper, we first present an overview of FBMC modulation and physical layer assumptions considered through our work. Consequently, we propose a medium access control (MAC) protocol for dense small cell networks operating in $5 \mathrm{GHz}$ unlicensed band. This MAC design is based on beacon enabled superframe similar to existing solutions $[3,4]$ with novel extension in order to support broadband traffic, multi-user channel access and to take advantage of FBMC signaling mainly to facilitate the resource allocation and channel access in uplink. In particular, the design is a mix of contention-based and scheduled-based access schemes that enable flexible use of radio resources and spectrum. Since the unlicensed $5 \mathrm{GHz}$ band is considered, the proposed MAC performs listen before talk (LBT) in order to comply with the ETSI regulatory requirements [5] and to ensure fair coexistence with neighboring technologies. In this band, two variants of channel access mechanisms are possible to comply with the regulation: frame-based equipment (FBE) and load-based equipment (LBE). LBE has been recently adopted by $3 \mathrm{GPP}$ within the licensed-assisted access (LAA) framework for component carriers in $5 \mathrm{GHz}$ band [6]. Similarly, in the proposed MAC design we rely on LBE as the channel access method used by the small cell to perform clear channel assessment (CCA) before transmitting. Second, we investigate the impact of PHY configuration parameters and MAC-related parameters on the performance of the proposed MAC design in dense deployment scenarios. Additionally, we analyze the fairness of coexistence of FBMC-MAC system when sharing the channel with a WiFi system.

The remainder of the paper is organized as follows. Section 2 describes the main features of FBMC modulation and physical layer assumptions. Section 3 presents the proposed MAC design and describes the frame structure as well as channel access modes. In section 4, the simulation results related to the PHYMAC designs are shown. Finally, the conclusions are given in section 5 .

\section{FBMC and PHY assumptions}

FBMC is a multi-carrier modulation considered as a $5 \mathrm{G}$ waveform candidate in order to address the OFDM limitations of reduced spectral efficiency and strict synchronization requirements [2]. Indeed, OFDM filters the sub-carriers using a rectangular pulse in the time domain leading to large side-lobes, thus important adjacent channel leakages. It requires the insertion of cyclic prefix $(\mathrm{CP})$ inducing a loss in the spectral efficiency. Moreover, OFDM suffers from high sensitivity to carrier frequency offset and high peak-to-average power ratio (PAPR) that limit uplink multi-user transmissions. In contrast, FBMC modulation uses a set of filters that synthesize and analyze each sub-carrier individually [7]. A proper design of the prototype filter can provide a very sharp spectral localization that allows multiple access and broadband data transmission.

The prototype filter is characterized by its overlapping factor $K$, which is the number of multi-carrier symbols that overlap in the time domain. The overlapping factor $K$ has an impact on determining the optimum spectrum utilization, 
the level of adjacent channel leakage (ACL) and the residual inter-symbol interference. In particular, offset quadrature amplitude modulation (OQAM) combined with Nyquist constraints on the prototype filter is considered to guarantee orthogonality between adjacent symbols and adjacent carriers while providing maximum spectral efficiency [2].

Figure 1 shows the power spectral density for OFDM and FBMC for several values of $K$. As it can be seen in the figure, a larger value of $K(K=4)$ is able to achieve better frequency localization and lower out-of-band emissions on adjacent channel $(<-100 \mathrm{dBc})$. At the contrary, for $K=2$, the leakage level increases significantly, and is only $10 \mathrm{~dB}$ lower than OFDM.

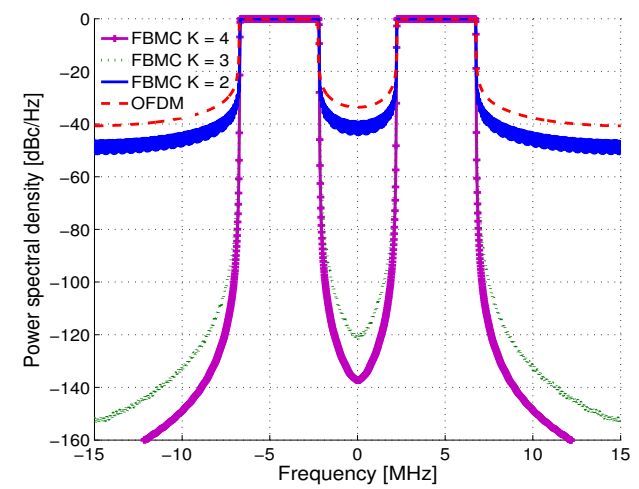

\begin{tabular}{ll} 
Parameter & 20 MHz band \\
\hline FFT size $N$ & 512 \\
Active carriers $N_{c}$ & 330 \\
Sub-carrier spacing $\Delta_{f}$ & $60 \mathrm{kHz}$ \\
overlapping factor $K$ & 4 \\
Bandwidth $B$ & $19.8 \mathrm{MHz}$ \\
Resource block $(\mathrm{RB})$ & $180 \mathrm{kHz}$ \\
Sampling Freq. $F_{s}$ & $30.72 \mathrm{MHz}$ \\
\hline
\end{tabular}

Fig. 1. Power spectral density of OFDM and FBMC with several values of $K$ (left) and FBMC parameters for a $20 \mathrm{MHz}$ carrier (right)

Compared to CP-OFDM, this excellent spectral localization of FBMC signal implies a set of characteristics that can exploited at MAC layer to optimize the medium access:

- Relaxed synchronicity: In CP-OFDM, simultaneous transmissions in contiguous bands have to respect a timing misalignment smaller than the $\mathrm{CP}$ length in order to avoid inter-carrier interference. By contrast, FBMC can gracefully tolerate asynchronous uplink communications on contiguous band, because of the very small level of adjacent carrier interference if a guard band of at least one sub-carrier spacing is introduced [8].

- Efficient use of allocated spectrum: In FBMC, the insertion of CP is not required and very low adjacent channel leakage ratio (ACLR) is achieved. Consequently, the MAC layer can manage in an efficient way the allocation of (thin) resource blocks, even if they are fragmented and spread in the band.

- Relaxed uplink power control: Along the same idea of very low ACLR, the uplink signal in contiguous bands can be transmitted without a strict power control. Indeed, provided that ACLR is more than $60 \mathrm{~dB}$, two simultaneous signals on contiguous channels can arrive on a small cell with a very large difference of uplink received power. 
In this work, the FBMC physical layer is assumed to rely on a particular implementation of FBMC called frequency spreading FBMC (FS-FBMC) according to the parameters specified in Figure 1 for $20 \mathrm{MHz}$ channel bandwidth. The total number of sub-carriers is set to 512 with sub-carrier spacing of $60 \mathrm{kHz}$ and overlapping factor of 4 . These parameters allow to reach the same level of performance of LTE-PHY with 2048 sub-carries and $15 \mathrm{kHz}$ sub-carrier spacing [8]. We note that the reduction of the number of sub-carriers by a factor of 4 reduces the PAPR which presents another advantage for uplink communication and allows also to reduce the complexity of FFT implementation. More details about the FBMC modulation and the filter coefficients can be found in [2], whereas implementation issues are presented in [9].

\section{FBMC-MAC protocol design}

This section presents the design of MAC protocol for $5 \mathrm{G}$ small cells to support broadband traffic operating in $5 \mathrm{GHz}$ unlicensed spectrum. Compared to the state of the art of PHY/MAC systems [3, 4], this design supports the use of FBMC physical layer, multiple access operation and incorporate LBT mechanisms to comply with ETSI regulatory requirements in the $5 \mathrm{GHz}$ band [5].

\subsection{MAC superframe design}

The MAC design is based on a beacon-enabled superframe, that specifies a combination of contention-based and scheduled-based access schemes close to the existing systems $[3,4]$. The basic network operation mode is a master-slave mode, with the small cell (SC) being the master of user equipments (UEs) within the radio range. More specifically, the superframe consists of a beacon period, a contention free period (CFP), a contention access period (CAP) and an idle period as illustrated in Figure 2. The length of the active superframe part is the channel occupancy time (COT) that is subdivided into multiple time slots, each slot has a duration of $1 \mathrm{~ms}$.

In the beacon period, the SC transmits beacon frames spanning on the whole band during one time slot. The beacon frames provide the basic timing for UEs synchronization and carry the control information for device discovery, network organization and resource allocations.

$\mathrm{CFP}$ is the scheduled access period composed of variable time slots that are especially allocated to downlink and uplink data communications. The allocation of resources is generally based on UE priority, QoS requirements and the availability of resources, the allocation being described in the beacon. We note that uplink data traffic is usually initiated by UE through sending an uplink grant request to the $\mathrm{SC}$ in a previous superframe, whereas downlink data traffic may be either requested by UE or initiated by the SC.

Meanwhile, the CAP is composed of small number of slots and used by UEs to send control and command frames such as association requests, ACK/NACK, 
channel quality indicator (CQI) reports and service establishment requests. On the other hand, control information, e.g. CQI, ACK, may be multiplexed with data in an uplink CFP slot when an active UE has both scheduled uplink and downlink traffic, thus allowing more flexibility in resource allocation.

As far as scheduled uplink traffic is concerned, the SC may configure an ACK slot at the end of the CAP in order to send grouped acknowledgments, if any, for the uplink received data in the CFP.

At the end of the superframe, an idle period is considered that can be advantageously used by SCs to sense the channel and feed the spectrum manager with sensing report accordingly.

It is interesting to note that the superframe structure can be dynamically adjusted depending on the traffic type and network load. Indeed, the length of CFP can be tuned to support a given traffic profile like a possible traffic imbalance between uplink and downlink. Alternatively, the CAP duration can also be tuned to provide a better reliability to ACK and CQI updates when the number of active UEs is getting large.

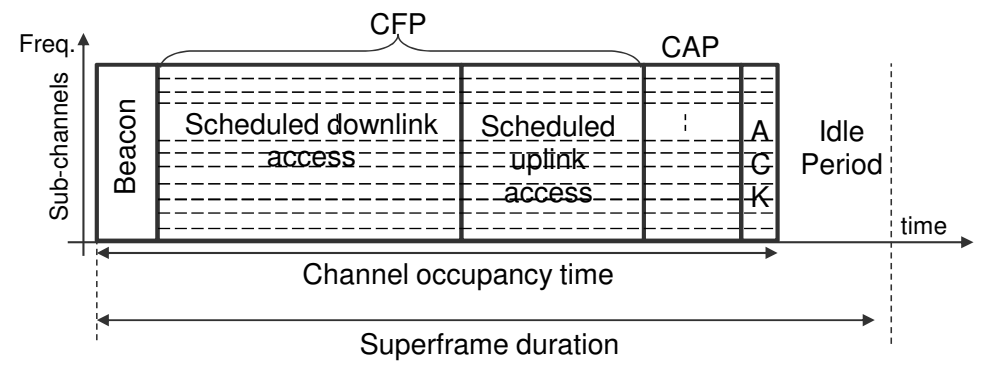

Fig. 2. MAC superframe structure for broadband access in $5 \mathrm{GHz}$

\subsection{Multiple access}

In the proposed FBMC-MAC design, multiple user access is supported both in CFP and CAP to allow efficient and dynamic share of the allocated spectrum.

In the CFP, multiple access is based on OFDMA-like approach for both uplink and downlink channels, using the same concept of resource block (RB) of LTE systems but applied to FBMC modulation. In FBMC-MA, a RB is an allocation of 3 active sub-carriers, i.e. $180 \mathrm{kHz}$, during a time slot. In this MAC, the scheduler is in charge of allocating both uplink and downlink resource blocks to active UEs, assuming the same kind of schedulers as those used in LTE. Interestingly, as FBMC tolerates asynchronous transmissions with the aforementioned guard interval constraint, uplink resource allocation can be done quite straightforwardly by the small cell using the same modulation scheme for both uplink and downlink communication. Figure 3 depicts the multiple access scheme on the superframe, for the scheduled access parts.

On the other hand, the multiple access in the CAP is based on multi-channel contention scheme. The CAP is therefore subdivided into several sub-channels, 
where each elementary sub-channel is composed of 17 active sub-carriers and one inactive sub-carrier as a guard band (6 resource blocks). Thus, 9 sub-channels and 18 sub-channels are assumed available for a system bandwidth of $10 \mathrm{MHz}$ and $20 \mathrm{MHz}$, respectively. UEs attempt to access the channel both in time and frequency domains using a multi-channel carrier sense multiple access with collision avoidance (CSMA/CA) algorithm [10]. Prior to transmission, UE first performs channel sensing to identify the set of idle sub-channels. If all sub-channels are busy, UE defers access until a sub-channel becomes available. Otherwise, UE selects one sub-channel and initiates a random back-off counter. Next, UE should perform CCA using a back-off procedure. If the channel is found busy during a back-off slot, UE may switch to another available sub-channel by maintaining the back-off counter. Otherwise, UE decrements the counter and transmits the frame when this counter reaches zero.

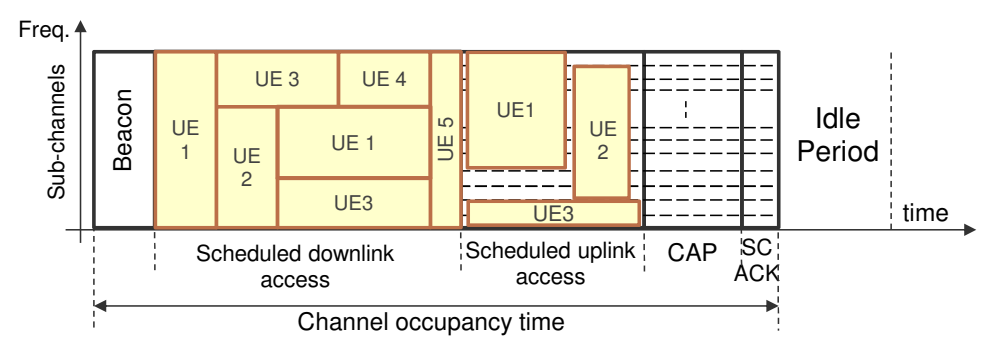

Fig. 3. Multiple access scheme in the scheduled part of the MAC superframe

\subsection{Shared spectrum utilization modes}

Since the operation in the $5 \mathrm{GHz}$ band is assumed, the $\mathrm{SC}$ has to comply with the LBT rule and shall perform a CCA procedure before the transmission of a MAC superframe to avoid interference with others systems. As stated before, the MAC design proposed in this paper can comply with the 2 access options specified by ETSI, which are namely FBE and LBE [5].

FBE defines a fixed timing frame for channel access, where the SC performs CCA in a fixed frame period. This access mode can induce unfair channel access in dense environment since a SC may get a definitive access to the channel and may block the activity of surrounding SCs.

In contrast, LBE relies on a flexible contention scheme prior to triggering the superframe emission that guarantees a better fairness in dense deployments. This channel access scheme has been retained for the LAA procedure in 3GPP specifications [6]. In our design, we have defined a set of parameters for LBE, based on the 3GPP specifications; they are shown in Figure 4. In this access mode, the SC performs an initial CCA over $m_{p}$ slot durations of a defer duration $T_{d}$. If the channel is found busy during one slot, the SC repeats the initial CCA. Otherwise, the SC shall perform an extended CCA (E-CCA) check over $N$ consecutive time periods of duration $T_{s} . N$ is randomly selected in the interval $[1, C W]$, where $C W$ is the length of the contention window. If an E-CCA turns 
out to be negative, i.e. channel is busy, an initial CCA is performed maintaining the current value of $N$. Otherwise, the $\mathrm{SC}$ decrements the counter $N$ and performs transmission of the superframe during COT when $N$ reaches zero. We assume that $\mathrm{COT}=10 \mathrm{~ms}$ and $T_{s}=9 \mu \mathrm{s}$. The LBE procedure is shown in Figure 4 for an example of $N=3$. More details about access priority of LBE access mode, the contention window size and the duration of the superframe can be found in $[6]$.

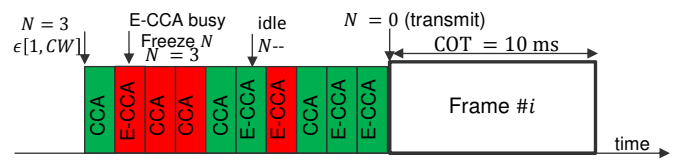

\begin{tabular}{ll} 
Access mode $T_{d}[\mu \mathrm{s}]$ & $C W$ \\
\hline $1 m_{p}=3$ & $16+9 \times m_{p}[15-63]$ \\
$2 m_{p}=7$ & $16+9 \times m_{p}[15-1023]$ \\
\hline
\end{tabular}

Fig. 4. LBE channel access for $N=3$ and access mode parameters

\section{Performance Evaluation}

In this section, we first describe the system-level simulation scenarios and various PHY/MAC parameters considered for assessing the performance of the proposed MAC design. Simulation results are presented and analyzed in a second step.

\subsection{Simulation assumptions}

We consider an outdoor deployment scenario based on regular hexagonal grid composed of 3 rings of $37 \mathrm{SCs}$, applying the wrap-around technique [11]. For each SC, we consider 10 UEs with distances to the SC being randomly distributed. The simulations are carried out in non-fading channel, using an extension of the Urban Micro (UMi) model [12], with spatial correlation of the LOS/NLOS and shadowing [11]. Several inter-site distances (ISDs) are considered to evaluate the performance of MAC design in different degrees of network densification and different energy detect (ED) thresholds ranging from $-62 \mathrm{dBm}$ to $-82 \mathrm{dBm}$ are considered to evaluate the impact of the CCA sensitivity with respect to densification. In terms of traffic, 100\% downlink full buffer pattern is considered as a worst case scenario. Adaptive modulation and coding (AMC) scheme is used by the SC similarly to LTE system based on CQI reports sent by UEs. Consequently, the SC assigns a suitable modulation and coding scheme (MCS) for each UE depending on channel conditions, that determines the transport block (TB) size to be transmitted on one slot. The interference between adjacent channels is modeled using out-of-band emissions masks for the considered underlying PHYs, e.g. FBMC and OFDM. For FBMC with $K=4$, no out-of-band emission is assumed. In case of OFDM and FBMC with $K=2$, the average of interference level on adjacent channel is assumed equal to $-37 \mathrm{dBc}$ and $-44 \mathrm{dBc}$ for $20 \mathrm{MHz}$ band, respectively. The main simulation parameters are summarized in Table 1.

In addition, two main coexistence scenarios are considered: non-coexistence and coexistence scenarios. In the non-coexistence scenario, we evaluate the performance of MAC design when it operates without any other interfering system. 
In case of the coexistence scenario, we focus on investigation the impact of WiFi system (operator B) on the performance of FBMC-MAC system (operator A) using the same $20 \mathrm{MHz}$ channel. In this case, we assume that WiFi APs are randomly dropped in the area with one AP per SC. WiFi uses standard distributed coordination function (DCF) CSMA/CA (without RTS/CTS signaling) with exponential back-off mechanism and transmission duration of $5 \mathrm{~ms}$ are assumed.

The performance metrics used in the evaluation are as follows: per-UE throughput, UE transmission delay, and channel occupancy statistics. Per-UE throughput is defined as the ratio of the number of correctly received bits over the given simulation time. The transmission delay is the averaged time required to successfully deliver a packet once it is at the head of the MAC queue. The mean channel occupancy is defined as the average of the total transmission time of SCs or WiFi APs over the simulation time, which is considered to evaluate the fairness of coexistence between FBMC-MAC and WiFi systems.

Table 1. Simulation parameters

\begin{tabular}{ll}
\hline - Parameter & Value \\
\hline Network Layout & Hexagonal grid, 1 sector by side \\
Inter-site distance (ISD) & 3 rings $/$ Wrap-around [11] \\
\hline Carrier frequency & $30 \mathrm{~m}, 50 \mathrm{~m}, 100 \mathrm{~m}$ \\
System bandwidth & $5 \mathrm{GHz}$ \\
Frequency reuse & $20 \mathrm{MHz}$ \\
\hline UE density & 1 and 3 \\
\hline Path loss model & $10 \mathrm{UEs}$ per SC randomly dropped \\
\hline Shadow fading model & Extended ITU-R UMi $[12]$ \\
\hline Channel fading model & UMi correlated log-normal shadowing $[12]$ \\
\hline SC Tx power & No fading \\
UE Tx power & $24 / 12 / 9 \mathrm{dBm}$ for ISD $=100 / 50 / 30 \mathrm{~m}$ \\
\hline SC/UE Antenna pattern & $20 / 12 / 8 \mathrm{dBm}$ for ISD $=100 / 50 / 30 \mathrm{~m}$ \\
SC/UE Antenna height & $2 \mathrm{D}$ Omni-directional \\
SC/UE Antenna Gain & $1.5 \mathrm{~m} / 10 \mathrm{~m}(\mathrm{ISD}=100,50 \mathrm{~m}) 6 \mathrm{~m}(\mathrm{ISD}=30 \mathrm{~m})$ \\
\hline SC/UE Noise Figure & $0 \mathrm{dBi} / 5 \mathrm{dBi}$ \\
\hline Traffic model & $9 \mathrm{~dB} / 5 \mathrm{~dB}$ \\
\hline Inter-cell interference model & Full Buffer $100 \%$ DL $($ no retransmission $)$ \\
\hline & Application packet size $=1500 \mathrm{Bytes}$ \\
Inter-channel interference model & Explicit \\
\hline & FBMC $K=4$, no leakage \\
& OFDM-LTE, ACL $=-37 \mathrm{dBc} / 20 \mathrm{MHz}$ \\
FBMC-MAC parameters & Scheduler: Round Robin \\
& CCA-ED $:-82 \mathrm{dBm},-62 \mathrm{dBm}$ \\
\hline & COT $=10 \mathrm{~ms}, m_{p}=3, \mathrm{CFP} / \mathrm{CAP}=6 / 3 \mathrm{slots}$ \\
\hline
\end{tabular}

\subsection{Simulation results}

Non-coexistence scenario In the non-coexistence scenario, the impact of inter-cell interference on the MAC performance is investigated assuming various system parameter settings. 
Figure 5 shows CDFs of per-UE throughput and UE delay of FBMC-MAC, given different ISDs and ED thresholds. It is observed that per-UE throughput decreases while transmission delay increases as the network is getting denser. This is due to the increase in UE density which leads to an increase of the offered load per $\mathrm{km} 2$ resulting in higher level of interference. This higher level of interference affects the channel quality leading to reducing the per-UE throughput and increasing the channel access delay. The impact of varying ED threshold is also depicted in Figure 5. The results show that lowering ED threshold from $-62 \mathrm{dBm}$ to $-82 \mathrm{dBm}$ improves per-UE throughput performance for dense scenarios (ISD $=30 \mathrm{~m}$ and $50 \mathrm{~m}$ ) due to the reduction of interference level and collision probabilities coming from the overall activity of cells. However, with ISD $=100 \mathrm{~m}$, this increase of ED sensitivity improves the throughput of cell-edge UE, still because of interference level reduction, while degrading the throughput of cell-center UE because more often SCs defer their transmissions. We notice that increasing ED thresholds results in reducing UE transmission delay, due to the increase of channel access opportunities of SCs.
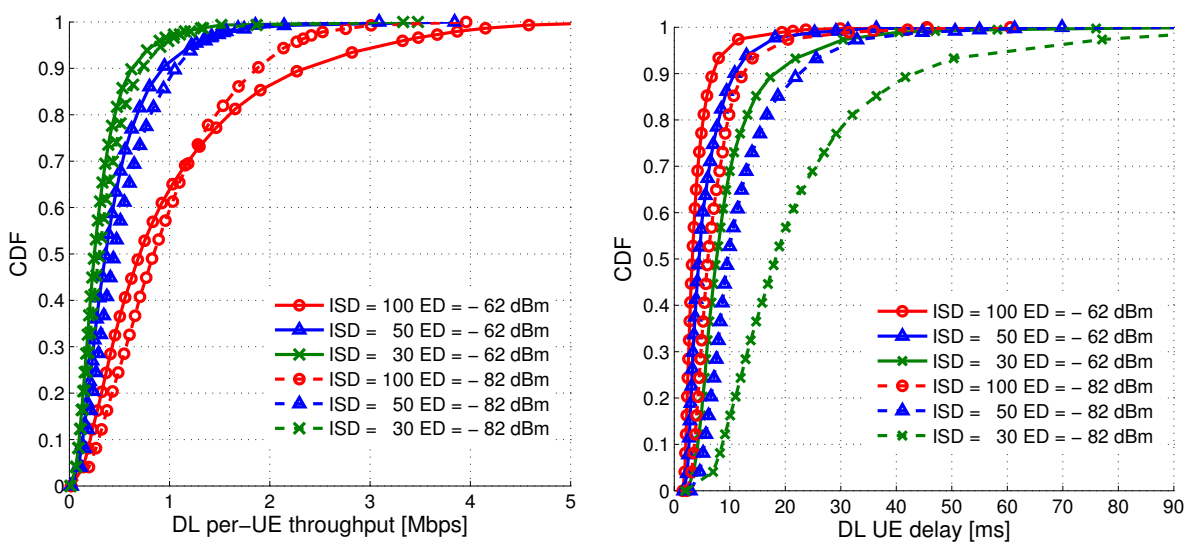

Fig. 5. CDFs of per-UE throughput (left) and transmission delay (right)

Figure 6 depicts per-UE throughput performance of FBMC with $K=4$, $K=2$ and ODFM using frequency reuse 3 , where adjacent SCs are assigned to different $20 \mathrm{MHz}$ channels. Comparing to the results with single channel in Figure 5 , it is observed that using a frequency reuse 3 significantly improves the performance as the contention for superframes is applied on more resource by the SCs. The increase of UE throughput is approximately 2.5 to 3 times higher compared to a single channel. The mean transmission delay is also significantly reduced as well as better channel access fairness is achieved. The impact of PHY modulation is illustrated in Figure 6. The results show that using FBMC with $K=2$ or OFDM reduces the throughput performance compared to FBMC $K=4$, especially for cell edge UEs. This stems from the adjacent channel interference caused by OFDM and FBMC with $K=2$, which affects the channel 
conditions as the level of interference is increased, this is particularly true for dense networks. Consequently, lower MCS will be assigned to UE data resulting in throughput degradation. The average UE throughput improvement of FBMC with $K=4$ compared to CP-OFDM is about $7.5 \%, 14 \%$ and $13 \%$ with ISD $=30$, 50 and $100 \mathrm{~m}$, respectively. In case ISD $=100 \mathrm{~m}$, the curves tend to reach a saturation throughput since the majority of UEs experience good channel conditions, and are assigned the higher MCS. It is also observed that there is no significant impact on UE delay since the traffic load in the system has not been changed regardless of adjacent channel leakages.
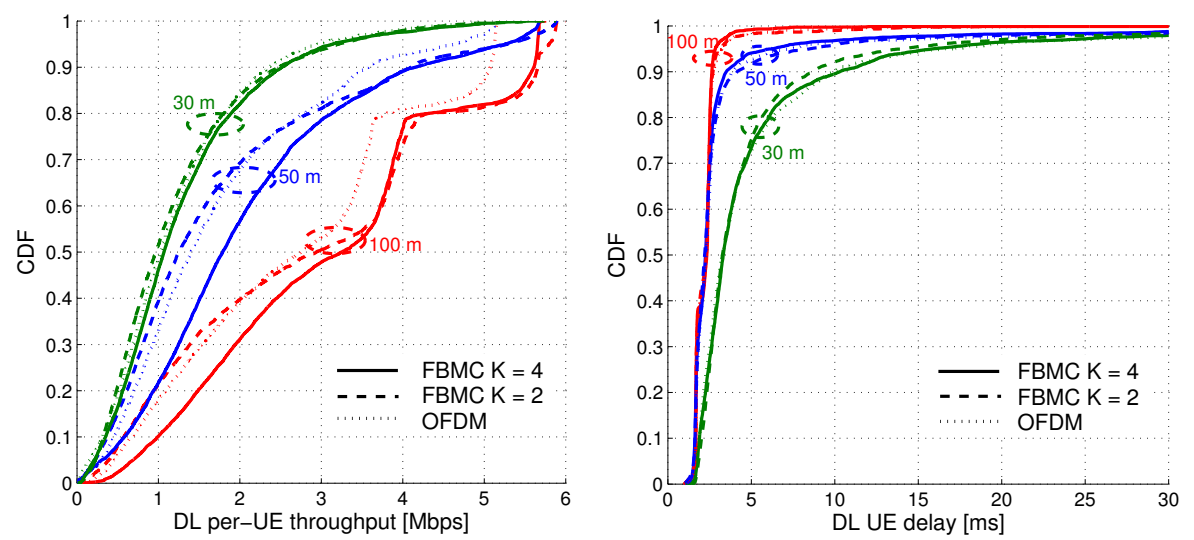

Fig. 6. CDFs of per-UE throughput (left) and transmission delay (right) using different PHYs (FBMC $K=4,2$, OFDM), ED $=-62 \mathrm{dBm}$, Frequency reuse 3

Coexistence scenario Herein we investigate the impact of WiFi system on FBMC-MAC performance and we evaluate the fairness of coexistence by comparing the mean channel occupancy of both systems.

Figure 7 shows the impact of WiFi on the per-UE throughput of FBMCMAC system and the impact on the channel occupancy of WiFi APs. Due to the shared channel nature and the existence of additional interference caused by WiFi system, we observe significant throughput degradation compared to non-coexistence scenario in Figure 5. We can see that lowering ED threshold to $-82 \mathrm{dBm}$ improves throughput performance and results in reducing the channel occupancy of WiFi system (Figure 8). Indeed, using an ED threshold of -62 dBm for both systems may result on severe interference and collision and degrade significantly the throughput performance of FBMC-MAC system.

The effect of using similar and asymmetrical ED thresholds of both systems is shown in Figure 8. The results show that using asymmetrical ED threshold improves the performance of one system at the expense of the other system whose activity is almost zero. On the contrary, using same ED threshold allows fair channel occupancy, in which better FBMC-MAC performance is achieved by lowering ED threshold of both systems to $-82 \mathrm{dBm}$ as shown in Figure 7. 

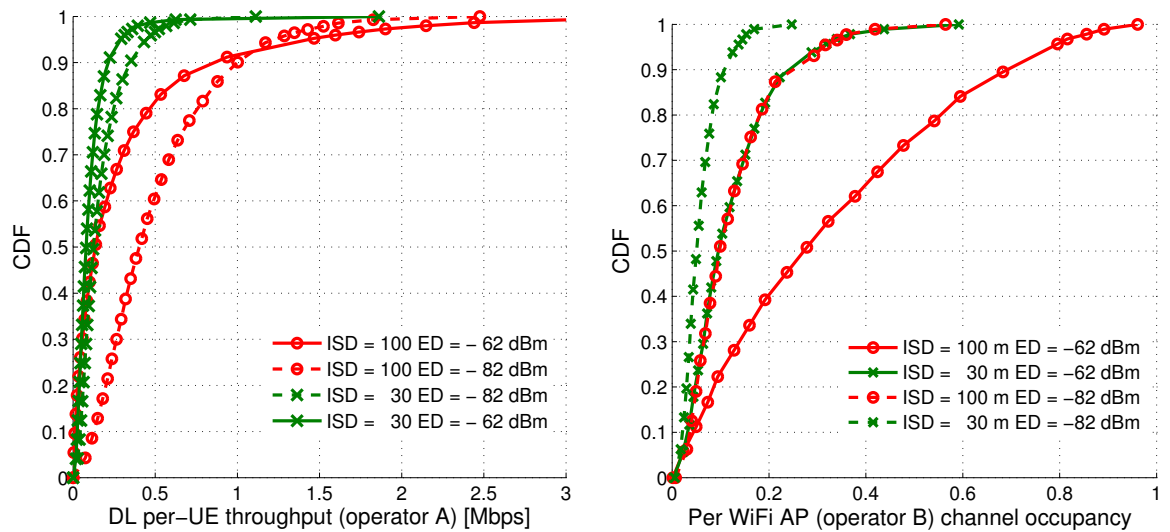

Fig. 7. CDFs of per-UE throughput for FBMC-MAC (operator A - left) and channel occupancy for WiFi APs (operator B - right)
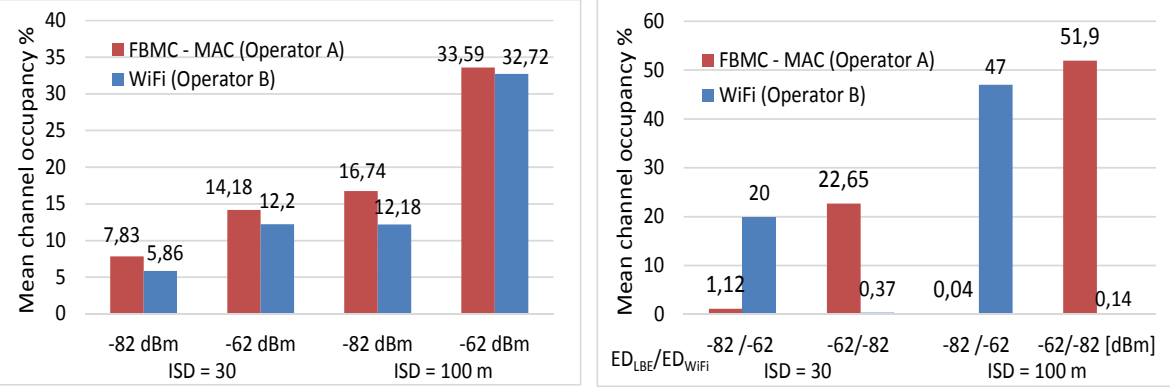

Fig. 8. Mean channel occupancy of FBMC-MAC (operator A) and WiFi (operator B), using similar ED threshold (left) and asymmetrical ED threshold (right)

Moreover, we have investigated the impact of FBMC-MAC (operator A) on the performance of WiFi (operator B) by considering that both operators deploy WiFi (i.e., FBMC-MAC SCs are replaced by WiFi APs). Although this is not shown in this paper, we have observed that the mean channel occupancy of operator B is similar if operator A deploys either WiFi or FBMC-MAC systems. It indicates that the LBT feature of FBMC-MAC is an efficient mechanism to provide fair coexistence with other systems operating in the same band.

\section{Conclusion}

In this paper, a MAC protocol for $5 \mathrm{G}$ small cells operating in $5 \mathrm{GHz}$ band has been presented. The proposed design exploits the benefit of FBMC modulation and allows asynchronous multi-user communications and efficient use of spectrum. It is based on beacon enabled superframe with scheduled-based and 
contention-based access schemes, in which superframe transmission is triggered by LBT procedure to coexist with others systems in the $5 \mathrm{GHz}$ band. The performance of the proposed FBMC-MAC design in case of non-coexistence and coexistence scenarios has been investigated for various network densities, physical layer configurations and LBT sensitivities. The results show that FBMCMAC design using well designed filter outperforms CP-OFDM systems in dense situations. We show also that FBMC-MAC design provides promising features for throughput improvement and fair coexistence with other systems (WiFi) through adjusting LBT thresholds. Future steps include further investigation of the performance of FBMC-MAC design, considering other network layouts (e.g. indoor hotspots), and others traffic models like uplink traffic where FBMC PHY features may provide additional gains compared to CP-OFDM systems.

\section{Acknowledgments}

The research leading to these results received funding from the European Commission H2020 program under grant agreement n671705 (SPEED-5G project).

\section{References}

1. NGMN: 5G White Paper, http://www.ngmn.org/home.html, July (2015)

2. M.Bellanger et al.: FBMC physical layer: a primer, http://www.ict-phydyas.org (2010)

3. ECMA 392 standard: MAC and PHY for Operation in TV White Space. Second edition, June (2012)

4. IEEE 1900.7-2015 standard: Radio Interface for White Space Dynamic Spectrum Access Radio Systems Supporting Fixed and Mobile Operation, December (2015)

5. ETSI EN 301893 V1.7.2, Broadband Radio Access Networks (BRAN): 5 GHz high performance RLAN; Harmonized EN covering the essential requirements of article 3.2 of the R\&TTE Directive, July (2014)

6. 3GPP TS 36.213, Evolved Universal Terrestrial Radio Access (E-UTRA), Physical layer procedures. Version 14.2.0, March (2017)

7. B. Saltzberg: Performance of an efficient parallel data transmission system. In: IEEE Trans. on Comm. Tech., vol. 15, no. 6, Dec. (1967)

8. R. Gerzaguet, et al: Comparative study of $5 \mathrm{G}$ waveform candidates for below $6 \mathrm{GHz}$ air interface. In: ETSI workshop on future radio technologies, Air interfaces, Sophia Antipolis, Feb. (2016)

9. Vincent Berg et al.: A flexible radio transceiver for TVWS based on FBMC. In: Microprocessors and Microsystems, Volume 38, Issue 8, pp. 743-753, Nov. (2014)

10. H. Kwon, H. Seo, S. Kim, and B. G. Lee: Generalized CSMA/CA for OFDMA systems: protocol design, throughput analysis, and implementation issues. In: IEEE Transactions on Wireless Communications, vol. 8, no. 8, pp. 4176-4187, Aug. (2009)

11. M. Filo, R. Edgar, S. Vahid, R. Taffazolli: Implications of wrap-around for TGax Scenario 3 and Scenario 4, Sept. (2015)

12. 3GPP TS 36.814: Evolved Universal Terrestrial Radio Access (E-UTRA), further advancements for E-UTRA physical layer aspects. Version 9.2.0, March (2017) 